\title{
Submillimeter vibrationally excited water emission from the peculiar red supergiant VY Canis Majoris
}

\author{
K. M. Menten ${ }^{1}$, S. D. Philipp ${ }^{1}$, R. Güsten ${ }^{1}$, J. Alcolea ${ }^{2}$, E. T. Polehampton ${ }^{3}$, and S. Brünken ${ }^{4}$
}

1 Max-Planck-Institut für Radioastronomie, Auf dem Hügel 69, 53121 Bonn, Germany e-mail: [kmenten;sphilipp;rguesten] @mpifr-bonn.mpg.de

2 Observatorio Astronomico Nacional, C/Alfonso XII, No. 3, 28014 Madrid, Spain e-mail: j . alcolea@oan.es

3 Space Science and Technology Department Rutherford Appleton Laboratory, Chilton, Didcot, Oxfordshire OX11 0QX, UK e-mail: E.T.Polehampton@rl.ac.uk

${ }^{4}$ Harvard-Smithsonian Center for Astrophysics, 60 Garden Street, Cambridge, MA 02138, USA e-mail: sbruenken@cfa.harvard.edu

Received 19 April 2006 / Accepted 23 May 2006

ABSTRACT

\begin{abstract}
Context. Vibrationally excited emission from the $\mathrm{SiO}$ and $\mathrm{H}_{2} \mathrm{O}$ molecules probes the innermost circumstellar envelopes of oxygenrich red giant and supergiant stars. VY CMa is the most prolific known emission source in these molecules.

Aims. Observations were made to search for rotational lines in the lowest vibrationally excited state of $\mathrm{H}_{2} \mathrm{O}$.

Methods. The APEX telescope was used for observations of $\mathrm{H}_{2} \mathrm{O}$ lines at frequencies around $300 \mathrm{GHz}$.

Results. Two vibrationally excited $\mathrm{H}_{2} \mathrm{O}$ lines were detected, a third one could not be found. In one of the lines we find evidence for weak maser action, similar to known (sub)millimeter $v_{2}=1$ lines. We find that the other line's intensity is consistent with thermal excitation by the circumstellar infrared radiation field. Several $\mathrm{SiO}$ lines were detected together with the $\mathrm{H}_{2} \mathrm{O}$ lines.
\end{abstract}

Key words. stars: AGB and post-AGB - stars: individual: VY Canis Majoris - supergiants - circumstellar matter

\section{Introduction}

In the past, millimeter-wavelength rotational lines from the lowest vibrationally excited state of water $\left(\mathrm{H}_{2} \mathrm{O}\right)$, the $v_{2}=1$ bending mode, have been detected from the innermost envelopes of oxygen-rich giant and supergiant stars. Menten \& Melnick (1989) used the IRAM $30 \mathrm{~m}$ telescope to discover the $v_{2}=1$, $J_{K_{\mathrm{a}}, K_{\mathrm{c}}}=5_{50}-6_{43}$ line near $232 \mathrm{GHz}$ toward the high mass-loss supergiant VY CMa and the nearby semiregular variable W Hya. Toward the former they also detected the $96 \mathrm{GHz} 440-533$ transition. Negative results were obtained for a number of other stars. All of the observed spectra contained a narrow (width $\approx 1 \mathrm{~km} \mathrm{~s}^{-1}$ ) feature, clearly indicating maser action. This feature also shows up in the spectrum of the $658 \mathrm{GHz} v_{2}=1,1_{10}-1_{01}$ ortho rotational ground-state transition, which has been found to show very strong maser action not only in VY CMa and W Hya, but in a variety of other (super)giant stars (Menten \& Young 1995). The excitation of these masers is discussed in Sect. 4.1.

There is also the interesting possibility that thermal emission from hot water has been detected: the $232 \mathrm{GHz}$ spectra of, both, VY CMa and W Hya show, in addition to the maser spike, broad emission over the velocity range covered by the circumstellar outflows of these objects.

The target of the observations is the peculiar red supergiant VY Canis Majoris. With a luminosity of $3.5 \times 10^{5} L_{\odot}$ (Sopka et al. 1985) and a mass-loss rate of $2-3 \times 10^{-4} M_{\odot} \mathrm{yr}^{-1}$ (Danchi et al. 1994) VY CMa is a remarkable object by any standard. For our discussions it is relevant to note that a plethora of maser lines from (ground-state and $v_{2}=1$ ) water and all the $\mathrm{Si}$ isotopes of silicon monoxide (from the $v=0$ to 5 states) have been detected from this object. At far-infrared wavelengths its spectrum is completely dominated by $\mathrm{H}_{2} \mathrm{O}$ lines including several lines from the $v_{2}=1$ state (Neufeld et al. 1999).

We used APEX to search for the $\mathrm{H}_{2} \mathrm{O} v_{2}=1,6_{61}-7_{52}$, $6_{60}-7_{53}$, and $5_{23}-6_{16}$ lines near 294,297 , and $336 \mathrm{GHz}$ are summarized in Sect. 2. In Sect. 3 we report the detection of the first and the third, but not of the second transition and present the observed spectra along with spectra of simultaneously observed silicon monoxide lines. After summarizing (in Sect. 4.1) our phenomenological picture of vibrationally excited $\mathrm{H}_{2} \mathrm{O}$ maser excitation, in Sect. 4.2 we investigate the possibility whether either or both of the detected lines may be the result of thermal excitation and come to the conclusion that this could be the case for one of them.

\section{APEX observations and data reduction}

Our observations were made in 2005 July/August under generally excellent weather conditions with the $12 \mathrm{~m}$ Atacama Pathfinder Experiment telescope (APEX ${ }^{1}$, see Güsten et al., this volume).

${ }^{1}$ This publication is based on data acquired with the Atacama Pathfinder Experiment (APEX). APEX is a collaboration between the Max-Planck-Institut für Radioastronomie, the European Southern Observatory, and the Onsala Space Observatory. 
Table 1. Water and silicon monoxide lines observed with APEX.

\begin{tabular}{|c|c|c|c|c|c|c|c|}
\hline $\begin{array}{l}\mathrm{H}_{2} \mathrm{O} v_{2}=1 \\
J_{K_{3} K_{c}}=\end{array}$ & $\begin{array}{l}\text { Frequency } \\
(\mathrm{MHz})\end{array}$ & $\begin{array}{l}E_{\ell} \\
(\mathrm{K})\end{array}$ & $\begin{array}{l}\int T_{\mathrm{MB}} \mathrm{d} v \\
\left(\mathrm{~K} \mathrm{~km} \mathrm{~s}^{-1}\right)\end{array}$ & $\begin{array}{c}v \text {-range } \\
\left(\mathrm{km} \mathrm{s}^{-1}\right)\end{array}$ & $\begin{array}{l}\Delta v \\
\left(\mathrm{~km} \mathrm{~s}^{-1}\right)\end{array}$ & $\begin{array}{l}T_{\mathrm{MB}} \\
(\mathrm{K})\end{array}$ & $\begin{array}{l}v_{\mathrm{LSR}} \\
\left(\mathrm{km} \mathrm{s}^{-1}\right)\end{array}$ \\
\hline $6_{61}-7_{52}$ & $293664.4^{a}$ & 3920 & $1.3(0.1)$ & $\sim[11,32]$ & $22(3)$ & 0.055 & $\sim 21$ \\
\hline $6_{60}-7_{53}$ & $297439.1^{a}$ & 3920 & $<0.16^{b}$ & - & - & $<0.029^{c}$ & \\
\hline $\begin{array}{l}5_{23}-6_{16} \\
J=8-7\end{array}$ & $336227.6^{a}$ & 2939 & $1.2(0.1)$ & $\sim[11,32]$ & $18(2)$ & 0.065 & $\sim 21$ \\
\hline${ }^{29} \mathrm{SiO} v=3$ & $335880.3^{d}$ & 5280 & $0.78(0.07)$ & {$[14,32]$} & $8.4(0.9)$ & 0.087 & $23.5(0.3)$ \\
\hline${ }^{30} \mathrm{SiO} v=1$ & $336602.4^{d}$ & 1804 & $41.3(0.5)$ & {$[-9,64]$} & $\sim 2^{e}$ & $3.1^{e}$ & $19.5^{e}$ \\
\hline${ }^{29} \mathrm{SiO} v=0$ & $342980.6^{d}$ & 58 & $34.2(0.3)$ & {$[-16,62]$} & $39.6(0.5)$ & 0.81 & $21.9(0.2)$ \\
\hline
\end{tabular}

Columns are (from left to right) quantum numbers of upper and lower state, frequency, energy above ground of lower state, integrated main-beam brightness temperature, velocity range covered by line (FWZP), FWHM line width from a Gaussian fit, peak main-beam brightness temperature, and centroid LSR velocity, Multiply $T_{\mathrm{MB}}(\mathrm{K})$ by 30 to get flux density in Jy. Numbers in parentheses are formal errors derived from Gaussian fitting and/or noise level analysis. ${ }^{a}$ Chen et al. (2000); ${ }^{b} 3 \sigma$ upper limit assuming a $20 \mathrm{~km} \mathrm{~s}^{-1}$ wide velocity range; ${ }^{c} 3 \sigma$ upper limit (in a spectrum smoothed to $1.5 \mathrm{~km} \mathrm{~s}^{-1}$ resolution); ${ }^{d}$ calculated from high precision molecular constants measured by Mollaaghababa et al. (1991); ${ }^{e}$ value for strongest velocity component.

The APEX 2 a facility receiver was used (Risacher et al., this volume), which covers the $278-375 \mathrm{GHz}$ frequency range that contains the frequencies of several $\mathrm{H}_{2} \mathrm{O}$ lines including the ones we observed. Two isotopic silicon monoxide lines were in the same band as the $336.2 \mathrm{GHz}$ line. A number of other lines were also observed including the ${ }^{29} \mathrm{SiO} v=0, J=8-7$ line. All lines considered here are listed in Table 1 and are discussed in Sect. 3. Calibration was obtained using the standard chopper wheel technique. The radiation was analyzed with the MPIfR Fast Fourier Transform spectrometer that provides 16384 frequency channels (Klein et al., this volume) over the $1 \mathrm{GHz}$ intermediate frequency bandwidth. To increase the signal to noise ratio, the spectra were smoothed to effective velocity resolutions appropriate for the measured linewidths, typically $1 \mathrm{~km} \mathrm{~s}^{-1}$. To check the telescope pointing the receiver was tuned to the $345.8 \mathrm{GHz}$ CO $J=3-2$ line. We measured 5 point rasters in azimuth and elevation centered on VY CMa itself and pointing corrections were determined by least squares fits. The pointing was found to be accurate to within $\sim 4^{\prime \prime}$, acceptable given the beam size, $\theta_{\mathrm{B}}$, which is $18^{\prime \prime} F W H M$ at $345.8 \mathrm{GHz}$. We present our line intensities in a main-beam brightness temperature $\left(T_{\mathrm{MB}}\right)$ scale and assumed a main-beam efficiency, $\eta_{\mathrm{MB}}$, equal to the observationally determined value at $345 \mathrm{GHz}$, i.e. 0.70 . We estimate that our intensity scale is accurate to within $15 \%$. The $F W H M$ beam size, $\theta_{\mathrm{B}}$, is $19^{\prime \prime}$ at $336 \mathrm{GHz}$ and $21^{\prime \prime}$ at $294 \mathrm{GHz}$.

\section{Results}

Figure 1 presents our observed spectra; see also Table 1 . We detect the two ortho- $\mathrm{H}_{2} \mathrm{O}$ lines $\left(5_{23}-6_{16}\right.$ and $\left.6_{61}-7_{52}\right)$, but not the latter line's para $\mathrm{H}_{2} \mathrm{O}$-equivalent $\left(6_{60}-7_{53}\right)$. Given that the 293 and $297 \mathrm{GHz}$ lines Einstein A-values and energies above ground are very similar, we would expect an intensity ratio of $\approx 3: 1$ for these lines if they were thermally excited. For the integrated intensities we obtain a lower limit of 8.2 for the ratio of the ortho to the para line, suggesting weak maser action that boosts the ortho line's intensity more than the para line's. The silicon monoxide lines are all between the $J=8$ and 7 rotational levels but within different vibrational states of vastly different energy above ground state. The ${ }^{30} \mathrm{SiO}, v=1$ line clearly shows maser action expressed in multiple emission spikes while all the other lines' profiles are consistent with (quasi)thermal emission. All lines are centered around $20 \mathrm{~km} \mathrm{~s}^{-1}$ which is within a few $\mathrm{km} \mathrm{s}^{-1}$ of the stellar velocity (whose exact value has an uncertainty of a few $\mathrm{km} \mathrm{s}^{-1}$ ). The velocity range covered by the ${ }^{29} \mathrm{SiO}, v=0$ line is widest and is comparable to that observed in other thermally excited lines of $\mathrm{SiO}$ and other molecules (e.g. Nyman et al. 1992). The $v=3{ }^{29} \mathrm{SiO}$ line and the two detected $\mathrm{H}_{2} \mathrm{O}$ lines are reasonably well fit by Gaussians. The very narrow width of $v=3{ }^{29} \mathrm{SiO}$ line $\left(\sim 8 \mathrm{~km} \mathrm{~s}^{-1}\right)$ indicates maser action. The $\mathrm{H}_{2} \mathrm{O}$ lines have about half the width of the ${ }^{29} \mathrm{SiO}, v=0$ line (and much poorer signal-to-noise ratio) and their shapes do not readily suggest masing, but, possibly, that they are formed in the innermost envelope where the stellar outflow has not yet reached its terminal velocity.

\section{Discussion}

\subsection{Vibrationally excited water masers}

Alcolea \& Menten (1993) noted that the $v_{2}=1$ maser lines described above seem to result from a systematic overpopulation of (what they call) the "transposed backbone" levels of the energy level diagram, (see their Fig. 1), i.e. levels with quantum numbers $K_{\mathrm{a}}=J$ and $K_{\mathrm{c}}=0$ and 1 for para- and ortho- $\mathrm{H}_{2} \mathrm{O}$, respectively, with respect to their neighboring levels. This situation is just opposite from that found in the ground state, where many of the "backbone" levels $\left(K_{\mathrm{a}}=0\right.$ or 1 and $\left.K_{\mathrm{c}}=J\right)$ may become overpopulated. This systematic inversion may be explainable by means of differential trapping in the radiative vibrational decays of the levels involved in those maser transitions and, possibly, by pumping to and decay from higher vibrational states. Detailed calculations to support this are still extant and not yet feasible, since collisional rates for vibrational transitions are unknown and can only be guessed (see Sect. 4.2).

The mechanism described above leads to the excitation of the known $4_{40}-5_{33}, 5_{50}-6_{43}$, and $1_{10}-1_{01}$ masers at 96, 232, and $658 \mathrm{GHz}$, respectively, and predicts inversion also, a.o., of the 294 and $297 \mathrm{GHz} 6_{61}-7_{52}$ and $6_{60}-7_{53}$ lines, of which we only detect the former one. However, given the uncertainties in our understanding of the maser excitation process it is by no means clear that these lines should be inverted at all. Certainly, there is no a priori reason to expect maser emission in the $336 \mathrm{GHz}$ line.

Given the spectral similarities between $\mathrm{SiO}$ maser lines and $v_{2}=1 \mathrm{H}_{2} \mathrm{O}$ lines pointed out by Menten \& Melnick (1991) it is highly likely that $v_{2}=1 \mathrm{H}_{2} \mathrm{O}$ maser emission arises from 


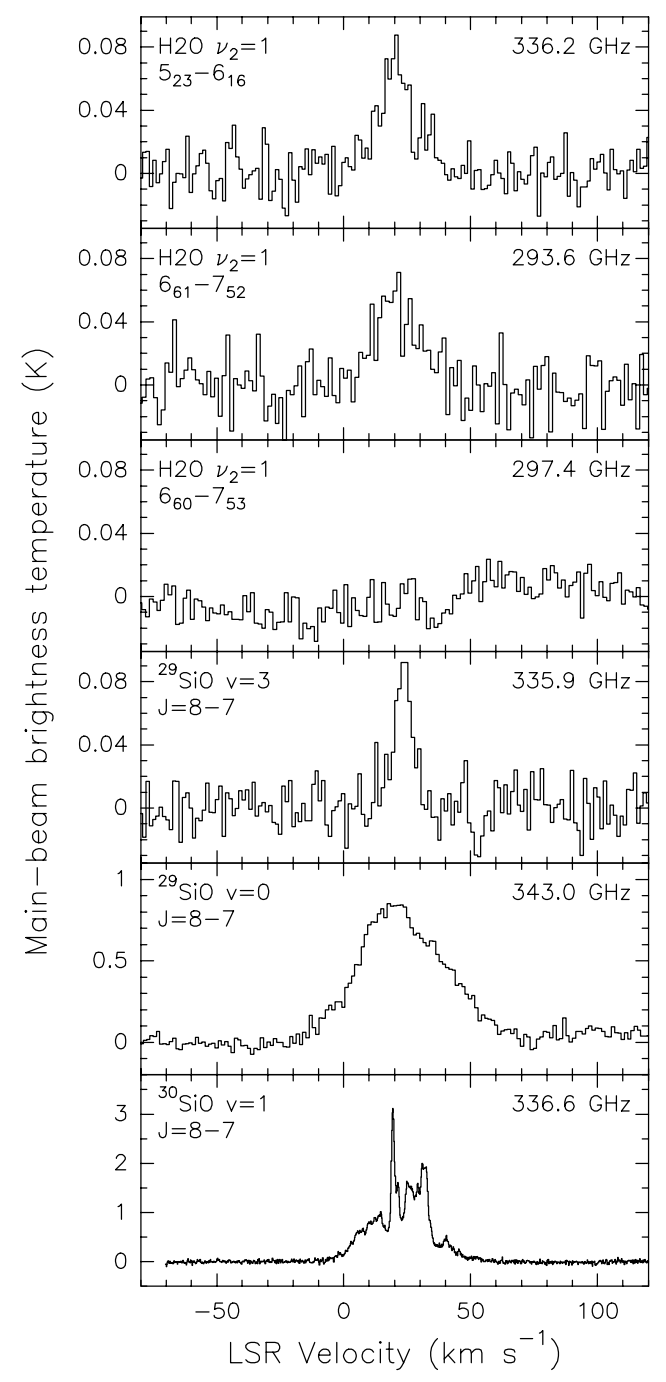

Fig. 1. Top to bottom: spectra observed toward VY CMA of the $\mathrm{H}_{2} \mathrm{O}$ $v_{2}=1,5_{23}-6_{16}, 6_{61}-7_{52}$, and $6_{60}-7_{53}$ lines and the $J=8-7$ lines of ${ }^{29} \mathrm{SiO}$ in the $v=3$ and 1 states and ${ }^{30} \mathrm{SiO}$ in the $v=1$ state. The spectra have been smoothed to channel spacings of 1.3, 1.5, 1.5, 1.3, 1.3, and $0.22 \mathrm{~km} \mathrm{~s}^{-1}$, respectively.

the same region as the multi-isotope/multi-v/multi- $J \mathrm{SiO}$ maser emission modeled by Gonzalez-Alfonso \& Cernicharo $(1997)^{2}$.

It may thus be expected that for some of the known $v_{2}=1$ lines part or all the emission is masing, although the excitation mechanism is still uncertain and may involve IR line overlaps. Could we, on the other hand, expect to observe thermal emission from a region of a size similar to that of the $\mathrm{SiO}$ maser region in lines unaffected by any inversion mechanism?

\subsection{The nature of the water lines detected with APEX}

To investigate whether the observed emission in one or both of the $\mathrm{H}_{2} \mathrm{O}$ lines detected by us might be of thermal nature, we make the following considerations. Assume that the line's level populations were in Local Thermodynamic Equilibrium (LTE), meaning $T_{\text {ex }}=T_{\text {rot }}=T_{\text {vib }}=T_{\text {kin }}$, i.e., that excitation, rotational,

\footnotetext{
${ }^{2}$ In particular, see Fig. 4 of Menten \& Melnick (1991), which shows that single tell-tale narrow $\left(\sim 1 \mathrm{~km} \mathrm{~s}^{-1}\right.$-wide), long lived ( $\left.>10 \mathrm{y}\right)$ feature at $22.3 \mathrm{~km} \mathrm{~s}^{-1}$ is seen in $\mathrm{SiO}$ maser lines from all three $\mathrm{Si}$ isotopes, as well as in both $v_{2}=1$ lines. In fact, strikingly, the $4_{40}-5_{33} \mathrm{H}_{2} \mathrm{O}$ and the ${ }^{29} \mathrm{SiO}$ and ${ }^{30} \mathrm{SiO} v=0, J=1-0$ lines show only emission in this feature.
}

and vibrational temperatures are all equal to the kinetic temperature in the emitting region and that the lines were thermalized, i.e. their level populations were described by a Boltzmann distribution at that temperature. If we assume a uniform medium, the line's main-beam brightness temperature, $T_{\mathrm{MB}}$, is given just by $f \times T_{\text {kin }}\left(1-\mathrm{e}^{-\tau}\right)$, where the beam-filling factor, $f$, is given by $\left(\theta_{\mathrm{S}} / \theta_{\mathrm{B}}\right)^{2} . \theta_{\mathrm{S}}$ and $\theta_{\mathrm{B}}$ are the $F W H M$ size of the Gaussian source and beam.

We now estimate whether plausible assumptions on the emission region could produce optically thick emission.

Around VY CMa, vibrationally ( $v=1$ and 2) SiO masers arise from a region of radius $\sim 0$. $^{\prime} 15$ (Menten \& Reid, in prep.). Assuming a distance of $1.5 \mathrm{kpc}$ for VY CMa (Lada \& Reid 1978 ), this corresponds to $3 \times 10^{15} \mathrm{~cm}$ or 20 stellar radii. Inside that region, $\mathrm{SiO}$ pumping models require a molecular hydrogen density, $n\left(\mathrm{H}_{2}\right)$, between $10^{9}$ and $10^{10} \mathrm{~cm}^{-3}$ (Lockett \& Elitzur 1992; Bujarrabal 1994).

In the following we assume a constant density of $2 \times$ $10^{9} \mathrm{~cm}^{-3}$ within $10^{15} \mathrm{~cm}$. This seems to be a plausible average value between $n\left(\mathrm{H}_{2}\right)=7 \times 10^{7} \mathrm{~cm}^{-3}$, the value Danchi et al. (1994) find for the inner radius of the dust shell and the density of $10^{12} \mathrm{~cm}^{-3}$ in the stellar photosphere; see, e.g., Reid \& Menten (1997). Danchi et al. derive $T=1563 \mathrm{~K}$ at the inner dust shell radius, while Le Sidaner \& Le Bertre (1996) and Massey et al. (2006) derive a temperature of 750-850 K for the dust from IR photometry. In the following we adopt $1000 \mathrm{~K}$ for the temperature. Assuming a homogeneous medium, we obtain an column density of $2 \times 10^{24} \mathrm{~cm}^{-2}$.

If we assume that the observed $v_{2}=1$ lines are thermalized we now can calculate their optical depths. For this we have used $A$-values and level energies from the HITRAN database (Rothman et al. 2003) and a value for the partition function, $Q(1000 \mathrm{~K})$, of 1194 from Chen et al. (2000). We use the upper range of the $\left[\mathrm{H}_{2} \mathrm{O} / \mathrm{H}_{2}\right]$ abundance ratios of $4 \times 10^{-4}$ that González-Alfonso \& Cernicharo (1999) derive for stars with high mass-loss rates while noting that Zubko et al. (2004) derive $4 \times 10^{-4}$ for VY CMa specifically in a completely independent analysis.

For $T=1000 \mathrm{~K}$ we compute optical depths of 1.0, 0.36, and 2.8 for the 294, 297, and $336 \mathrm{GHz}$ lines, respectively, and $T_{\mathrm{MB}}$-values of 12,5 , and $28 \mathrm{mK}$. The lower value of the $297 \mathrm{GHz}$ para-line as compared to the $294 \mathrm{GHz}$ ortho-line is due to the 3:1 ortho-to-para ratio.

The calculated value for the $336 \mathrm{GHz}$ lines is only a factor of 2.3 lower than the actually measured intensity, while that ratio for the $293 \mathrm{GHz}$ line is larger, $\sim 5$. For the broad component of the $232.6 \mathrm{GHz} 5_{50}-6_{43}$ line detected by Menten \& Melnick (1989) with a $12^{\prime \prime} F W H M$ beam we calculate $T_{\mathrm{MB}}=43 \mathrm{mK}$ for $T=1000 \mathrm{~K}$, which is smaller than the $140 \mathrm{mK}$ they observe. As discussed in Sect. 4.1 one might expect inversion for both of the latter lines and the weak maser action they do indeed seem to show (see Sect. 3) can explain their intensities.

The above estimates assume that our $\mathrm{H}_{2} \mathrm{O}$ lines are thermalized. Thermalization can either be achieved by collisional excitation or radiative excitation. To check the potential of collisional excitation, we calculate the lines' critical densities. The critical density of a transition from level $i$ to level $j$ is given by $\sum A_{i j} / \sum \gamma_{i j}$, where the sums are over all possible transitions originating from level $i . A_{i j}$ are the Einstein $A$-coefficients (again from HITRAN) and $\gamma_{i j}$ the collisional rate coefficients, calculated by Green et al. (1993) for different $T$-values and accessed online via the Leiden Atomic and Molecular Database (Schöier et al. 2005). Rate coefficients for collisions from the ground to the $v_{2}=1$ state are unknown. In the following we assume them 
to be identical to transitions within the ground state with identical $J, K_{\mathrm{a}}$ and $K_{\mathrm{c}}$ quantum numbers multiplied by a factor $c_{\mathrm{vg}}$; the study of González-Alfonso et al. (2002) suggests $c_{\mathrm{vg}}=0.02$. For all of our three $v_{2}=1$ transition we calculate $\sum A_{i j}=21 \mathrm{~s}^{-1}$ within $10 \%$. The sums of the collisional rate coefficients for the three lines are very similar as well: we calculate (within 5\%) $\sum \gamma_{i j}=c_{\mathrm{vg}} 2.1 \times 10^{-10} \mathrm{~cm}^{3} \mathrm{~s}^{-1}$ for $1000 \mathrm{~K}$. From this we derive $n_{\text {crit }}=c_{\mathrm{vg}}^{-1} \times 10^{11} \mathrm{~cm}^{-3}$. Comparing this with the densities discussed above $\left(\sim 10^{8}-10^{10} \mathrm{~cm}^{-3}\right)$ we find that our lines are very unlikely to be thermalized by collisions in the whole $10^{15} \mathrm{~cm}$ radius region.

As to radiative excitation: according to Carroll \& Goldsmith (1981), in a region with a strong IR field the criterion for radiative excitation to determine the populations of energy levels of a rotational line that is coupled by IR radiation of frequency $v_{\mathrm{IR}}$ to levels in an excited vibrational state is $f /\left(\mathrm{e}^{h \nu_{\mathrm{IR}} / k T_{\mathrm{S}}}-1\right)>$ $A_{\text {rot }} / A_{\text {vib }}$. Here $A_{\text {vib }}$ and $A_{\text {rot }}$ are the $A$-values the rotational and vibrational transitions involved, respectively, and $T_{\mathrm{S}}$ is the effective temperature of the IR source. For our case the filling factor, $f$, is equal to 1 and $v_{\mathrm{IR}} \sim 4.5 \times 10^{4} \mathrm{GHz}$ (corresponding to $6.7 \mu \mathrm{m}$ ), which results in the ratio in left hand side of the equation to being $\approx 0.2$. This is much larger than the right hand side, since $A_{\text {vib }} \sim 20 \mathrm{~s}^{-1}$ and $A_{\text {rot }} \sim 10^{-6} \mathrm{~s}^{-1}$. Given this, it seems safe to assume that the level populations of the submillimeter lines are thermalized at the temperature of the emitting region.

In summary, the level populations of the observed $\mathrm{H}_{2} \mathrm{O}$ $v_{2}=1$ lines are likely to be excited via infrared radiation. Making simple, but plausible assumptions on their dense, hot emission region, we can roughly reproduce the observed intensity of the $336 \mathrm{GHz}$ line assuming thermal excitation. Known characteristics of $\mathrm{H}_{2} \mathrm{O} v_{2}=1$ masers lead one to expect that the 294 and $297 \mathrm{GHz}$ lines might have inverted populations. Indeed, the observed intensity of the $294 \mathrm{GHz}$ ortho-line suggests that it might be boosted by weak maser action. The non-detection of its twin para- $\mathrm{H}_{2} \mathrm{O} 297 \mathrm{GHz}$ line is naturally explained by the lower abundance of the para species and less amplification. Future imaging with ALMA, whose highest resolution will resolve the lines' emission region, will yield important information on this higly interesting environment.

\section{References}

Alcolea, J., \& Menten, K. M. 1993, Astrophysical Masers, LNP, 412, 399 Bujarrabal, V. 1994, A\&A, 285, 971

Carroll, T. J., \& Goldsmith, P. F. 1981, ApJ, 245, 891

Chen, P., Pearson, J. C., Pickett, H. M., Matsuura, S., \& Blake, G. A. 2000, ApJS, 128,371

Danchi, W. C., Bester, M., Degiacomi, C. G., Greenhill, L. J., \& Townes, C. H. 1994, AJ, 107, 1469

Gonzalez-Alfonso, E., \& Cernicharo, J. 1997, A\&A, 322, 938

González-Alfonso, E., \& Cernicharo, J. 1999, ApJ, 525, 845

González-Alfonso, E., Wright, C. M., Cernicharo, J., et al. 2002, A\&A, 386, 1074

Green, S., Maluendes, S., \& McLean, A. D. 1993, ApJS, 85, 181

Lada, C. J., \& Reid, M. J. 1978, ApJ, 219, 95

Le Sidaner, P., \& Le Bertre, T. 1996, A\&A, 314, 896

Lockett, P., \& Elitzur, M. 1992, ApJ, 399, 704

Massey, P., Levesque, E. M., \& Plez, B. 2006, ArXiv Astrophysics e-prints

Menten, K. M., \& Melnick, G. J. 1989, ApJ, 341, L91

Menten, K. M., \& Melnick, G. J. 1991, ApJ, 377, 647

Menten, K. M., \& Young, K. 1995, ApJ, 450, L67

Mollaaghababa, R., Gottlieb, C. A., Vrtilek, J. M., \& Thaddeus, P. 1991, ApJ, 368, L19

Neufeld, D. A., Feuchtgruber, H., Harwit, M., \& Melnick, G. J. 1999, ApJ, 517, L147

Nyman, L.-A., Booth, R. S., Carlstrom, U., et al. 1992, A\&AS, 93, 121

Reid, M. J., \& Menten, K. M. 1997, ApJ, 476, 327

Rothman, L. S., Barbe, A., Benner, D. C., et al. 2003, JQSRT, 82, 5

Schöier, F. L., van der Tak, F. F. S., van Dishoeck, E. F., \& Black, J. H. 2005, A\&A, 432, 369

Sopka, R. J., Hildebrand, R., Jaffe, D. T., et al. 1985, ApJ, 294, 242

Zubko, V., Li, D., Lim, T., Feuchtgruber, H., \& Harwit, M. 2004, ApJ, 610, 427 